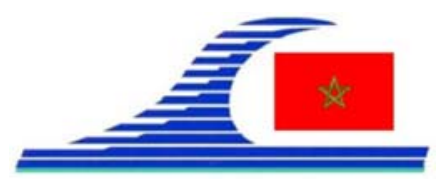

Conférence Méditerranéenne Côtière et Maritime

EDITION 2, TANGER, MAROC (2011)

Coastal and Maritime Mediterranean Conference

Disponible en ligne - http://www.paralia.fr - Available online

\title{
Les digues sous-marines en géotextiles pour la protection des plages : avantages et inconvénients
}

\author{
Pierre FARNOLE ${ }^{1}$, Julie LEBUNETEL ${ }^{1}$
}

1. ERAMM, 1503 rte des Dolines, BP 42, 06901 Sophia Antipolis, France.

pf@eramm.fr ; jl@eramm.fr

\section{Résumé :}

On observe actuellement une augmentation sensible à l'échelon national et international des ouvrages de protection contre l'érosion marine ayant un caractère de "réversibilité" d'une part et présentant d'autre part un moindre coût par rapport à des techniques de protection classiques en enrochements. Il s'agit dans la plupart des cas d'ouvrages en forme de tubes constitués d'une double enveloppe en géotextile rempli de sable. De longueur et de hauteur variables les tubes sont installés dans les petits fonds en amas ou en couple parallèlement à la côte pour provoquer le déferlement des vagues et réduire l'érosion des plages. Cette technique est actuellement très prisée et de nombreuses applications ont vu le jour au cours de cette décennie: Plage de la Capte à Hyères ; plage de Young-Jin Beach en Corée du Sud et plus récemment à Moriani plage en Haute Corse et sur la plage de la Croisette à Cannes. Le retour d'expérience sur certaines de ces applications est présenté dans cet article en particulier la tenue à la mer des ouvrages et leur efficacité contre l'érosion marine.

Mots-clés :

Tubes en géotextile - Sédiments - Protection du littoral - Digues sous-marines - Epis

\section{Principe}

L'utilisation des géotextiles pour la protection du littoral suit les techniques déjà mise en place pour les protections classiques en enrochements (brise-lames, épis, perrés). Considérée comme une solution légère, économique et réversible (KOFFLER et al., 2009), elle consiste à pomper du sable à l'aide d'une drague et à remplir les tubes posés sur un tapis anti affouillement. Le remplissage des tubes se fait au niveau des cheminées raccordées à la pompe dont le débit doit être contrôlé et selon un protocole préétabli. Cette étape très délicate doit tenir compte des caractéristiques du géotextile, de la granulométrie et du comportement physique du sédiment injecté dans le tube. L’objectif consiste à atteindre une forme de l'enveloppe qui réponde au dimensionnement préétablit.

\section{Les contraintes de remplissage}

Le taux de remplissage a un impact sur le comportement et la forme du tube. En fonction de ces paramètres, les efforts hydrodynamiques auxquels l'ouvrage est soumis 
La connaissance de la Mer :

un vecteur du développement durable en Méditerranée

et sa réaction aux efforts seront différents. Il est établi que le pourcentage de remplissage est un paramètre fondamental pour la stabilité du tube (RECIO \& OUMERACI, 2006 ; 2007). Plus le taux de remplissage est faible, plus le tube sera instable. En effet, un remplissage excessif aura tendance à donner une forme quasicirculaire et une rigidité accrue car l'enveloppe géotextile sera en traction réduisant ainsi la mobilité des sédiments à l'intérieur du tube. En cas de remplissage insuffisant, le tube aura tendance à s'étaler en prenant une forme ellipsoïde. Le tube sera plus souple et sa capacité à la déformation augmentera, affectant sa stabilité. La plus grande difficulté lors de la mise en place du tube réside dans le maintien d'une cote altimétrique et de la forme préalablement définie. En effet, une fois rempli, le tube est susceptible de se tasser par une évacuation différée de l'eau contenue dans la suspension ; phénomène qui a été déjà observé lorsque les pores du géotextile sont colmatés par des grains de petite taille. La perméabilité du géotextile est alors compromise et l'eau se trouve piégée à l'intérieur du tube, ce qui a pour conséquence une modification générale de la forme au fur et à mesure que le trop plein d'eau s'évacue.

\section{Le dimensionnement}

De manière générale, le dimensionnement des tubes s'effectue en termes de géométrie de l'ouvrage (longueur, largeur et hauteur) et en fonction des contraintes hydrodynamiques auquel il doit résister. Outre les difficultés liées au remplissage, le dimensionnement de ce type d'ouvrages est délicat car ce sont des structures qui évoluent au cours du temps, en fonction des conditions hydrodynamiques, des caractéristiques du textile et du matériau de remplissage. La forme du tube à un instant $\mathrm{t}$ dépend de l'historique des houles dont il a subi l'influence (RECIO \& OUMERACI, 2006 ; 2007). A chaque tempête, la forme du tube est susceptible d'être modifiée créant potentiellement et ponctuellement un problème de stabilité qui se traduit par une déformation (torsion) et parfois par des déchirures. De plus, sous certaines conditions (forçage, granulométrie, saturation en eau), des phénomènes de liquéfaction des sédiments peuvent se manifester affectant ainsi la stabilité du tube. Malgré une large utilisation des géotextiles dans le domaine maritime, depuis une trentaine d'années et ce, de part le monde, il n'existe pas, à notre connaissance, de formulations adaptées à chaque configuration de protection prenant en compte l'ensemble des contraintes de ces structures.

\section{Quelques applications}

\subsection{Plage de la Capte à Hyères}

Depuis le printemps 2008, deux sections de $100 \mathrm{~m}$ et $150 \mathrm{~m}$ ont été installées par des fonds de -1,8 m accompagnée d'un rechargement de plage avec $12000 \mathrm{~m}^{3}$ (KOFFLER 
et al., 2009 ; MEULE et al., 2010). Cette opération a donné satisfaction car elle a stabilisé la plage en réduisant l'énergie de la houle à la côte et la fuite des sédiments. Après trois années d'exploitations, les tubes nécessitent des réparations car ils ont subi des déchirures importantes avec des cheminées arrachées et un tassement général de la digue est observé. Les travaux d'entretien consistent à réparer les déchirures et à poser de nouveaux tubes sur ceux existants pour surélever l'ouvrage.

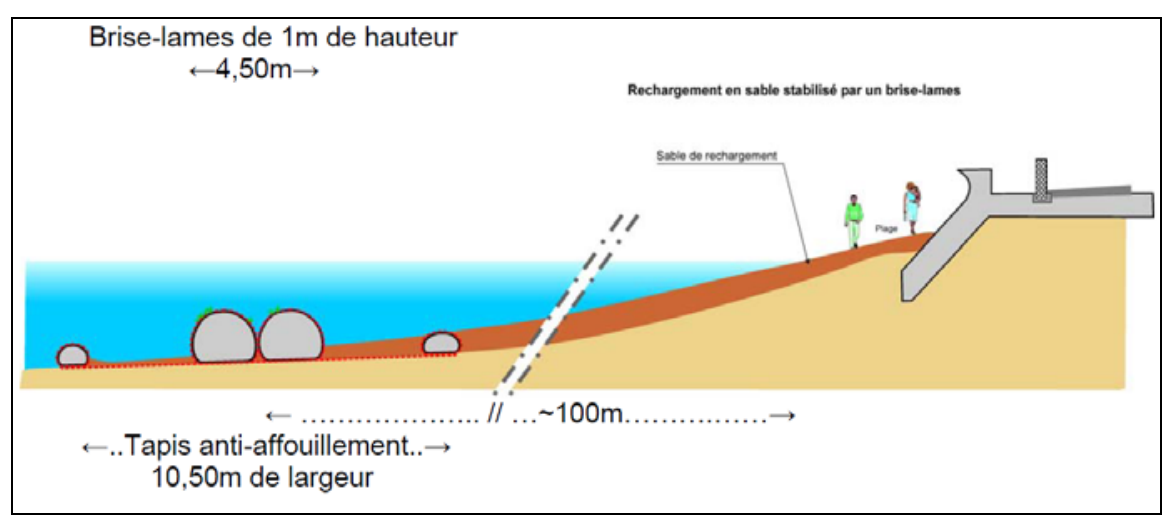

Figure 1. Schéma d'implantation des tubes à la Capte (KOFFLER et al., 2009).

\subsection{Moriani plage (Haute-Corse)}

Ces travaux réalisés au printemps 2010 ont consisté à installer des 5 épis en géotextile de 40 à $50 \mathrm{~m}$ de longueur espacé de $60 \mathrm{~m}$. Le géotextile est en polypropylène avec double enveloppe capable de résister aux UV et à la houle. Le remplissage s'est fait à terre avec un sable de $600 \mu \mathrm{m}$. Après une année d'exploitation, les résultats sont globalement satisfaisants puisque les tubes ont stabilisé la plage et ont résisté aux intempéries de l'hiver. On notera un ensouillement en tête d'épi et des phénomènes de torsion du tube induit par l'action des vagues déferlant sur l'ouvrage.
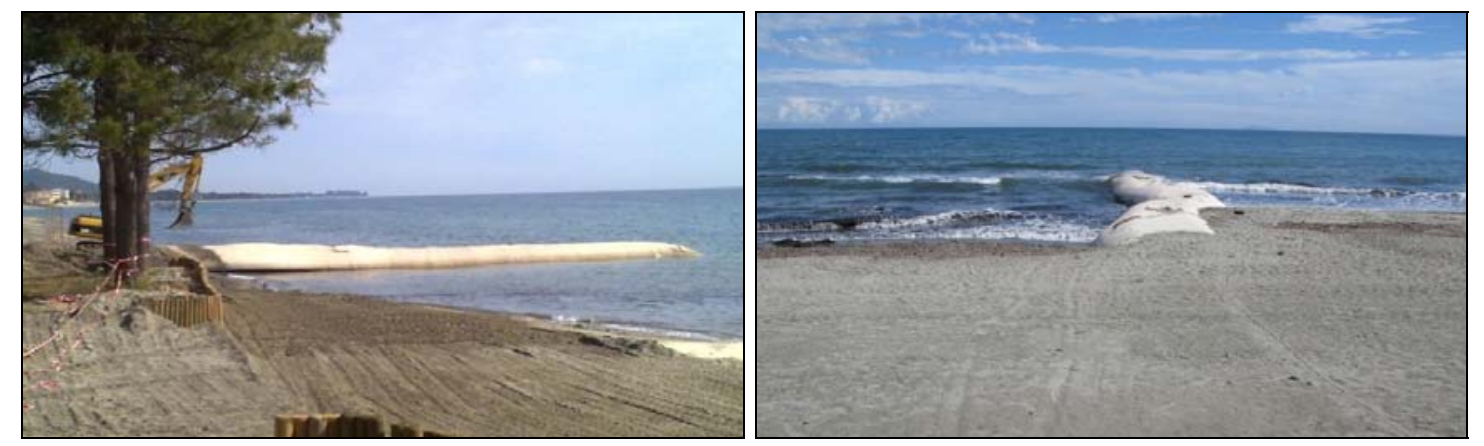

Figure 2. Prises de vues des épis à Moriani Plage.

\section{Conclusions}

Les tubes en géotextiles installés sur les plages subissent des désordres à plus ou moins brèves échéances (déchirure, torsion, ...) qui oblige les maîtres d'ouvrage à procéder à 
La connaissance de la Mer :

un vecteur du développement durable en Méditerranée

des travaux d'entretien sans quoi l'ouvrage serait à terme détruit. Des progrès sensibles ont été réalisés par les fabricants de géotextile qui fournissent maintenant des produits hautement résistant ce qui assure une durée de vie plus longue des ouvrages. Cependant, les agressions physiques en dehors des efforts hydrodynamiques sont bien souvent à l'origine des déchirures (hélices de bateau, bois morts, vandalisme, ...). S'agissant des efforts hydrodynamiques, le caractère déformable de ces structures en liaison avec la capacité mobilisatrice du sédiment de remplissage rend délicat leur dimensionnement. En ce qui concerne leur efficacité pour la protection des plages, celle-ci a été démontrée dans plusieurs cas, à condition de disposer au préalable d'une bonne connaissance des processus hydrosédimentaires et des causes du phénomène d'érosion mais aussi à condition que l'ouvrage résiste dans la durée. S’agissant des coûts, ils sont de l'ordre de $150 \mathrm{~K} €$ pour un épi compris entre 50 et $100 \mathrm{~m}$ et $200 \mathrm{~K} €$ pour un brise-lame immergé sur un même intervalle. La réduction du coût par rapport à des ouvrages en enrochements serait de 3 pour un épi et de 5 pour un brise-lame. Cette technique a un bel avenir car elle a peu d'effet négatif sur le milieu naturel et paysager. Mais à l'heure actuelle, l’ouvrage en géotextile doit être considéré comme un ouvrage de protection temporaire pour la protection du littoral.

\section{Références bibliographiques}

RECIO J., OUMERACI H. (2006). Processes Affecting the Stability of Revetments made with Geotextile Sand Containers, Abstract for ICCE Conference 2006.

RECIO J., OUMERACI H. (2007). Effect of deformations on the hydraulic stability of coastal structures made of geotextile sand containers. Geotextiles and Geomembranes, Volume 25 (4-5), pp 278-292. doi:10.1016/j.geotexmem.2007.02.006

KOFFLER A., ZENGERINK E., ASCIONE J.-C., BIRUKOFF J.-M. (2009). Un atténuateur de houles en tube géosynthétique pour limiter l'érosion de la plage de la Capte à Hyères. Rencontres géosynthétiques 2009.

MEULE S., PAQUIER A., CERTAIN R., BOUCHETTE F., GRATIOT J., SABATIER F., ROBIN N. (2010). Morphodynamique de la plage de la capte, Hyères, Var, suite à la mise en place d'atténuateurs de houle en géotextile. XI ${ }^{\text {èmes }}$ Journées Nationales Génie Côtier - Génie Civil, Les Sables d’Olonne, pp 369-378. doi:10.5150/jngcgc.2010.044-M 